CARNETS DE Carnets de géographes

GÉOGRAPHES.

$5 \mid 2013$

Géographie humanimale

\title{
Les animaux sauvages au village
}

Entre sources de conflits et attractions touristiques (Bornéo, Sabah, Malaysia)

\section{Clotilde Luquiau}

\section{(2) OpenEdition}

Journals

Édition électronique

URL : http://journals.openedition.org/cdg/1068

DOI : $10.4000 / \mathrm{cdg} .1068$

ISSN : 2107-7266

Éditeur

UMR 245 - CESSMA

Référence électronique

Clotilde Luquiau, «Les animaux sauvages au village », Carnets de géographes [En ligne], 5 | 2013, mis en ligne le 01 janvier 2013, consulté le 10 décembre 2020. URL : http://journals.openedition.org/cdg/ 1068 ; DOI : https://doi.org/10.4000/cdg.1068

\section{(c) $\Theta \Theta \Theta$}

La revue Carnets de géographes est mise à disposition selon les termes de la Licence Creative Commons Attribution - Pas d'Utilisation Commerciale - Pas de Modification 4.0 International. 


\title{
LES ANIMAUX SAUVAGES AU VILLAGE \\ Entre sources de conflits et attractions touristiques (Bornéo, Sabah, Malaysia)
}

\author{
CLOTILDE LUQUIAU \\ Université Paris Ouest Nanterre \\ Doctorante en géographie, \\ Centre Asie du Sud-Est, UMR 8170 \\ clotilde.luquiau@ymail.com
}

\section{Résumé}

Cet article traite du tourisme animalier de vision dans le "sanctuaire " faunistique de la plaine alluviale de la Kinabatangan, une zone protégée du nord de Bornéo, en Malaysia. Fondé sur des données collectées au gré de plusieurs séjours entre 2006 et 2012, il explore la question de la reconnaissance de l'animal comme acteur. Le récit et la mise en scène de la rencontre avec l'animal (non-humain) sont mis en perspective grâce à la prise en compte du rôle de l'homme dans l'espace dit « naturel » et à l'analyse de la concurrence entre acteurs pour l'organisation de ce territoire. L'observation de la zone protégée montre que la place de la faune est en permanente négociation et que le tourisme animalier opère un glissement spatial en requalifiant l'espace liminaire entre forêt et terroir villageois. Le travail de terrain démontre que la liberté de déplacement de la faune est reconnue par les acteurs pour servir leurs propres intérêts. La fréquentation des bordures du " sanctuaire " par les animaux est un vecteur de conflits avec les sociétés locales mais elle compose également le cœur du produit touristique.

\section{Abstract}

This article is about wildlife watching tourism in the Lower Kinabatangan Wildlife Sanctuary of the state of Sabah, Malaysia. It is based on a research project through which data was collected during several fieldtrips from 2006 to 2012. It aims at assessing the potential for recognising the "agency" of animals. It also explores the concept of "humanimal collective" in order to qualify the existing relationship between human society and local fauna in Lower Kinabatangan. The narrative and the staging of the encounter of humans and (non-humans) animals is put into perspective through the analysis of human interactions with the so-called "wilderness" and through the examination of competition for the organisation within this territory. Observations carried out in respect to the protected area reveal that wildlife habitat is continuously being negotiated. It also shows that wildlife-tourism is in itself the 
precursor of a spatial shift that now causes a requalification of the space that exists between the village and the forest. Indeed, animals increasingly frequent the margins of the sanctuary and this is creating conflicts with the local people. And yet wildlife is the core of the tourism product. This article argues that animals are given more liberty when it serves the interests of the stakeholders.

\section{Introduction}

Le tourisme animalier de vision consiste en la rencontre pacifique entre êtres humains et animaux « sauvages » dans leur habitat « naturel ». Dans l'état malaisien ${ }^{1}$ du Sabah, au nord de Bornéo (figure 1), le sanctuaire faunistique de la basse Kinabatangan (figure 2) a été créé pour protéger l'habitat d'orangs-outans, d'éléphants pygmées, de singes nasiques, de calaos, etc. Habitants et touristes ne peuvent pas pénétrer dans cette zone sans autorisation spéciale. Malgré les clôtures électriques et les chenaux de drainages des plantations alentours (figure 3), la faune reste libre de se déplacer hors de la zone protégée. Cette autonomie représente un atout pour la mise en scène du produit touristique mais une menace pour les agriculteurs. Pour assurer le succès du produit touristique, les animaux doivent fréquenter l'espace liminaire entre la zone protégée et l'espace habité et cultivé: le bord du fleuve Kinabatangan, les rives des étangs et surtout les abords des hôtels. Le tourisme participe à la protection de l'environnement en lui apportant une justification économique mais tours opérateurs et environnementalistes ont des conceptions différentes de la nature et de l'espace à protéger (Dalla Bernardina, 1996). Les premiers défendent la préservation d'un paysage à forte densité de macrofaune tandis que les seconds visent la protection d'un habitat assurant la pérennité des espèces menacées, quitte à ce qu'elles ne soient plus visibles.

La faune est à la fois un produit touristique, un patrimoine constitutif de la biodiversité et une nuisance. Sa place est donc en perpétuelle négociation. Quels sont les enjeux sociaux et spatiaux de la mise en tourisme d'un espace protégé, dans lequel la rencontre avec l'animal est à la fois facilitée par la conservation, recherchée par les touristes et évitée par les habitants?

Cet article prend source dans une recherche portant sur les liens entre développement, protection de l'environnement et tourisme dans la plaine de la Kinabatangan. De 2006 à 2011, une cinquantaine d'entretiens formels et informels ont été menés auprès d'acteurs clés de la conservation, du tourisme et de l'administration du Sabah, 118 questionnaires ont

\footnotetext{
${ }^{1}$ En français, le terme Malaisie se réfère soit à la péninsule (Malaya), soit à la fédération de Malaisie (Malaysia).Le terme Malaysia est préféré ici car il désigne sans ambuiguité la fédération de Malaisie qui englobe les territoires du Sarawak et du Sabah ainsi que l'île de Labuan.
} 
été effectués auprès de touristes et 160 auprès des habitants. Ces données sont complétées par une observation participante, d'abord en tant que touriste puis en tant que géographe enquêtrice hébergée chez l'habitant et enfin comme guide accompagnatrice d'un groupe de touristes francophones. Des courriels ont été exploités pour compléter les données.

Après avoir situé notre position scientifique, nous montrons en quoi la logique de conservation s'articule avec la mise en tourisme du territoire. Ensuite, nous analysons les modalités des conflits entre la société locale et la faune pour explorer la notion de territoire et de collectif humanimal (Baratay, 2010). Enfin, nous montrons que la mise en scène de la nature sauvage sert avant tout l'intérêt économique des opérateurs et assure la satisfaction des touristes.

\section{L'animal, un acteur de l'écosystème}

Une partie des opérations de conservation de la zone protégée de la basse Kinabatangan est financée par la vente d'œuvres peintes par les animaux du zoo de Houston. Cette opération est, à bien des égards, emblématique. Le site du zoo présente un à un ses pensionnaires en les nommant, en décrivant leur caractère et leurs techniques d'artistes. "Parmi les plus productifs des artistes (...), Cheyenne [une femelle orang-outan], peint avec ses doigts mais en général, elle manie son pinceau d'une façon caractéristique en traçant des rayures d'un côté à l'autre. Parfois, elle ajoute de l'eau avec sa bouche en créant une technique de peinture mixte ". Cette description présente un primate agissant suivant son inspiration, individualisé des autres et réalisant un produit culturel. Dans le tourisme animalier de vision, nombreux sont ceux qui adhèrent cette conception des animaux.

II n'est pas question de trancher le débat entre les comportementalistes, qui considèrent que les bêtes n'agissent qu'en réponse à des stimuli pour satisfaire leurs besoins, et les défenseurs de l'éthologie cognitive qui soutiennent que l'animal est un sujet capable d'apprendre, possédant une culture (Lestel, 2001: 10). Les stratégies des animaux nous viennent de la description faite par les scientifiques et les habitants. Force est de constater que nos informateurs décrivent la faune, surtout les primates et les éléphants, en les dotant d' " intelligence ", de " liberté ». Des scientifiques affirment qu'ils possèdent une " culture » (Van Schaik \& Ancrenaz, 2003). Ce champ lexical rejoint la notion d'agentivité, qui désigne la capacité d'agir de façon intentionnelle et imprévisible d'un sujet démontrant des capacités d'apprentissage et de réflexivité. Nous admettons ici que les non-humains peuvent être considérés comme des "actants" (Lussault, 2007) et même comme des acteurs, dans la mesure où ils peuvent transformer le système auquel ils appartiennent (Baratay, 2010 ; Mounet, 2007 :18). En effet, les animaux ne se confinent pas à l'espace qui leur est assigné par les hommes. L'incursion d'une nouvelle espèce comme le loup en Vanoise, bouleverse l'ordre préexistant en favorisant la création de réseaux entre des mondes auparavant fermés (Mauz, 2005). 
Figure 1 : Les principales aires protégées du Sabah

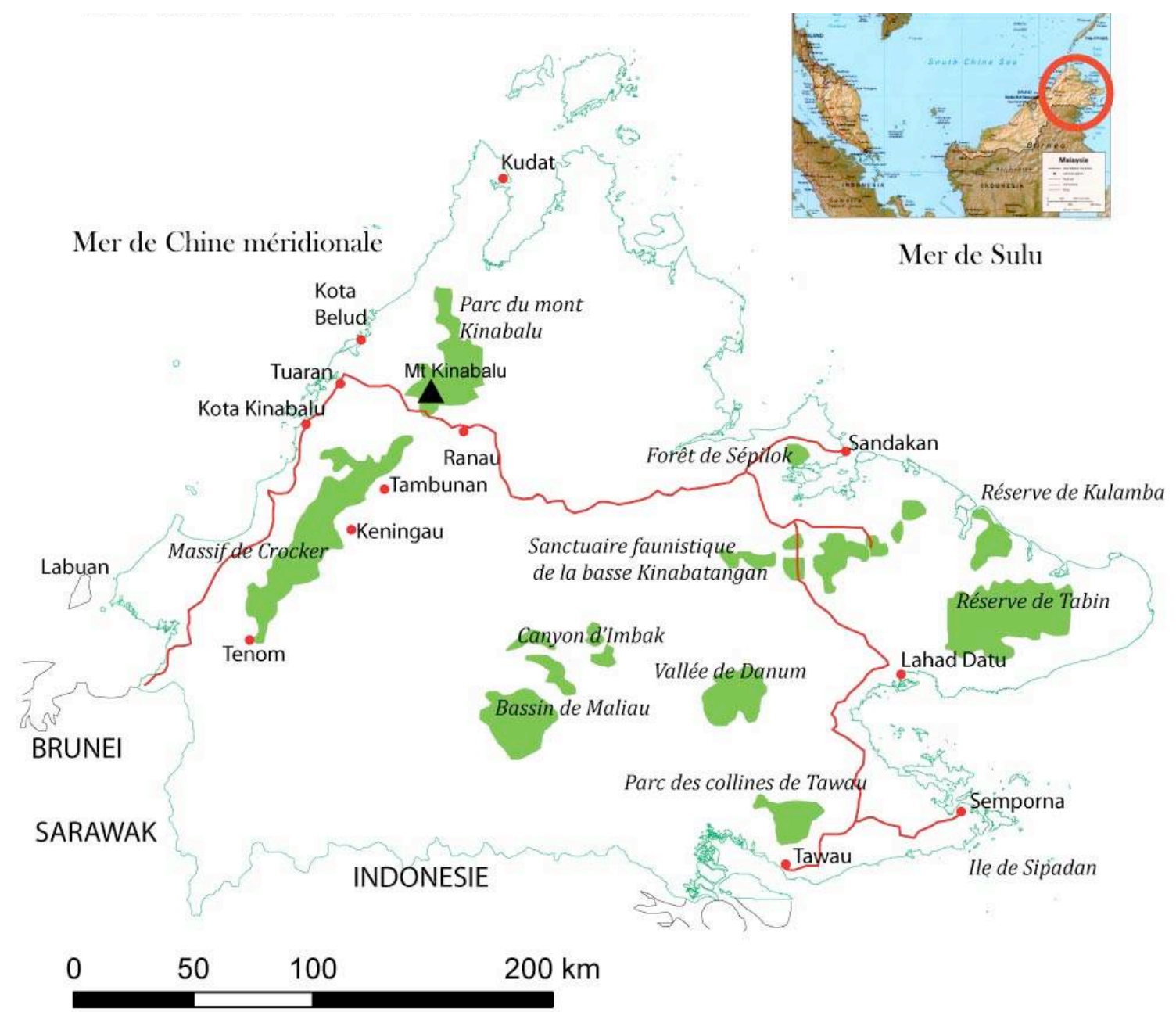

Réalisation : Clotilde Luquiau 2012

Source: Sabah Forestry department 2007, Wendy Hutton 2002

Le succès du tourisme animalier de vision dans la basse Kinabatangan: la requalification de l'espace animal

Le tourisme animalier désigne la visite d'une faune non domestiquée dans son habitat « naturel » ou reconstitué, en captivité ou non, dans le cadre de la plongée, la chasse, la pêche ainsi que la visite de parcs zoologiques. L'engouement pour ce secteur résulterait du "besoin de nature " ressenti par les classes urbaines (Estebanez, 2006). Selon les estimations de Higginbottom, le tourisme animalier représente 20 à $40 \%$ du tourisme et 600 millions de personnes visitent des parcs où les animaux sont en captivité. Les zones protégées totalisent 12\% des terres émergées (Higginbottom, 2004). En général, ce sont à la fois des aires récréatives pour les touristes qui s'affranchissent des normes sociales de leur vie professionnelles (Mowforth et Al., 2008) et des aires contraignantes pour les habitants 
dont les usages quotidiens sont encadrés et subordonnés à la conservation de la nature (Dalla Bernardina, 1996).

La "nature sauvage » est une notion construite, un espace idéalisé où l'anthropisation apportée par les peuples locaux, peu visible, est oubliée, voire niée par l'utilisation de termes tels que "vierge ", " primaire ", " intouchée » (Héritier, 2008 ; Mowforth \& Munt, 2008 ; Michaud 2011 b; Mittman, 1999). Cette représentation conduit parfois à l'éviction de peuples autochtones (Gunnell, 2009 :173). Et pourtant, les travaux d'anthropologues, de géographes et d'agronomes prouvent que les sociétés humaines participent à la richesse de la biodiversité (Dove et Al., 2005) et tout particulièrement les habitants de Bornéo (Padoch et Al., 2003 ; Sellato, 1998 ; De Koninck, 2007).

Avec la "reforestation ", il est même évident que l'homme participe à la reconstitution d'écosystèmes artificiels. Les degrés de "naturalité » des paysages sont variables (Godet, 2010) et l'appréciation du caractère naturel d'un lieu dépend d'un ensemble de représentations (Michaud, 2011 a; Zeppel \& Muloin, 2008 :117) : là où les scientifiques voient une forêt secondaire dégradée, l'habitant voit les vergers plantés par ses ancêtres et le touriste accompagné par un guide peu scrupuleux est invité à admirer une forêt vierge.

Le tourisme animalier introduit de nouveaux acteurs : les opérateurs touristiques et leurs clients qui veulent admirer les animaux. Comme le montre Isabelle Mauz, chaque groupe d'acteurs a sa conception de leur place: pour les habitants, la place des espèces domestiques est proche de la maison, celle des espèces "sauvages » est dans les endroits éloignés et même inaccessibles (Mauz, 2002). Au contraire, dans une logique touristique, pour des raisons d'accessibilité, la place idéale de la faune est dans l'espace liminaire entre l'espace fréquenté par les touristes et l'espace "naturel » où a lieu la rencontre hommeanimal. Il est donc préférable que la population animale soit dense pour augmenter sa visibilité. Les opérateurs "vendent" en effet un paysage constitué d'une végétation d'apparence naturelle et peuplée d'une faune photogénique. En pratique, le secteur touristique bénéficie donc des contraintes qui restreignent l'habitat animal.

Grâce à sa forêt tropicale, ses fonds marins et la relative qualité de ses infrastructures, le Sabah vient régulièrement en tête des destinations du tourisme animalier d'Asie (Revolinski, 2012). Les sites phares sont la réserve de forêt "primaire " de Danum Valley, la fosse marine de l'île de Sipadan, le centre de réhabilitation des orangs-outans de Sepilok, le parc du mont Kinabalu et le sanctuaire de la basse Kinabatangan (figure 1). Cette dernière est l'objet d'un tourisme animalier de vision concernant une faune sauvage en liberté dans une zone protégée et officiellement non-clôturée. 
Figure 2 : Le projet de couloir écologique présenté par WWF Malaysia en 2001

NB : Le sanctuaire est constitué des parcelles vert clair, la surface jaune est le couloir que WWF appelle à reboiser. Il est constitué de villages et de plantations. C'est majoritairement là que les conflits entre la faune et les humains sont rapportés.

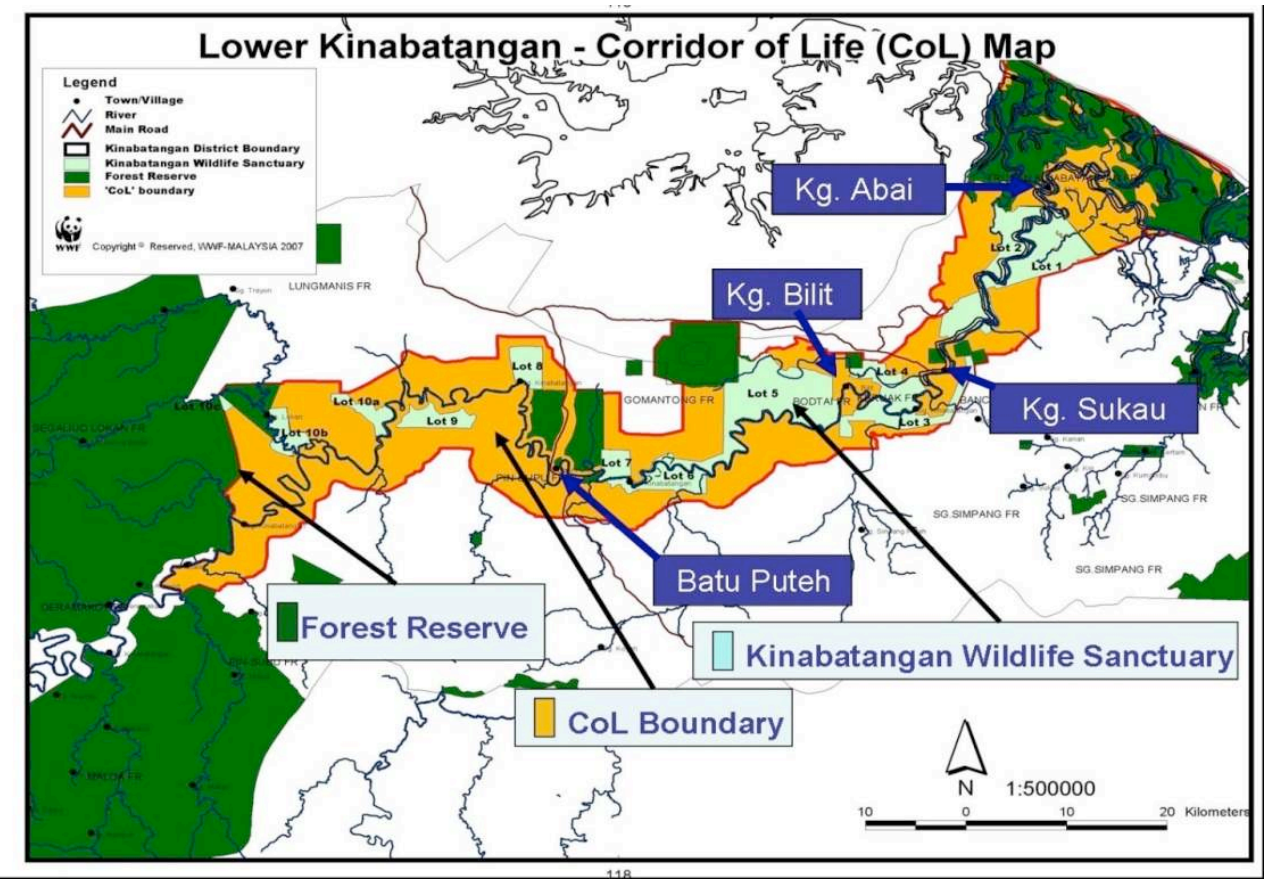

Une dizaine de villages sont situés à la lisière du sanctuaire. Les habitants, nommés Orang Sungai (gens de la rivière) possèdent en général un petit potager attaché à leur maison et de petites parcelles de palmiers à huile. Anciens chasseurs-cueilleurs semi-itinérants vivant principalement du commerce des nids d'hirondelles jusque dans les années cinquante, puis de la coupe du bois, ils tirent aujourd'hui leurs revenus de petites plantations de palmiers à huile, de la pêche et de modestes activités liées à l'économie résidentielle. Abai, Sukau, Bilit et Batu Puteh, totalisant environ 500 foyers, sont les quatre villages disposant d'hébergements touristiques marchands et d'un homestay: une association d'habitants proposant des chambres aux touristes.

Les hôtels, nommés lodges, sont une quinzaine et offrent près de 400 chambres. II y aurait environ 50000 touristes par an dans la partie touristique de la Kinabatangan, de Sungei Lokan à Abai (figure 2). Même si les retombées directes sont limitées, le tourisme offre désormais des revenus complémentaires à quelques villageois qui travaillent dans les lodges, aux propriétaires qui louent une parcelle aux opérateurs touristiques et enfin aux soixante-dix familles des homestays (estimations 2010).

Cette forme de community based tourism a été accompagnée par les ONG environnementales locales: le Fond Mondial pour la Nature (WWF-Malaysia), présent au Sabah depuis les années 80 (Payne 1989) et l'ONG Hutan, créée en 1997 par deux Français, 
une biologiste, Isabelle Lackman et un vétérinaire, Marc Ancrenaz (ainsi que d'autres ONG et bailleurs de fond qui eux, ne sont pas implantés directement dans le sanctuaire). Enfin, Hutan et Danau Girang Field Center, la station scientifique liée à l'université de Cardiff, ouverte en 2008, accueillent chercheurs et étudiants. Les ONG et les scientifiques travaillent en collaboration avec le Sabah Forestry Department et avec le Wildlife Department, luimême rattaché au Ministry of Tourism, Culture and Environment Sabah, comme en témoignent les publications communes et les projets cofinancés (Ambu \& Al., 2012).

En 2005, les réserves forestières bordant la Kinabatangan et gérées par le Sabah Forestry Department, sont transformées en wildlife sanctuary. Le "sanctuaire " est constitué de parcelles boisées disjointes totalisant 26100 ha (Sabah State, 2005) (figure 2). Il est coupé en son sein par deux routes, les habitations et le fleuve. La protection de la jungle locale établit un rempart plus sûr à l'extension des plantations de palmier à huile. Cela sécurise les tours opérateurs existants et attire de nouveaux concurrents. La loi les oblige à choisir des parcelles jouxtant les zones habitées ce qui valorise les terrains situés entre villages et forêt.

Figure 3 : carte des obstacles au passage des éléphants (légende traduite d’Estes et Al., 2012)

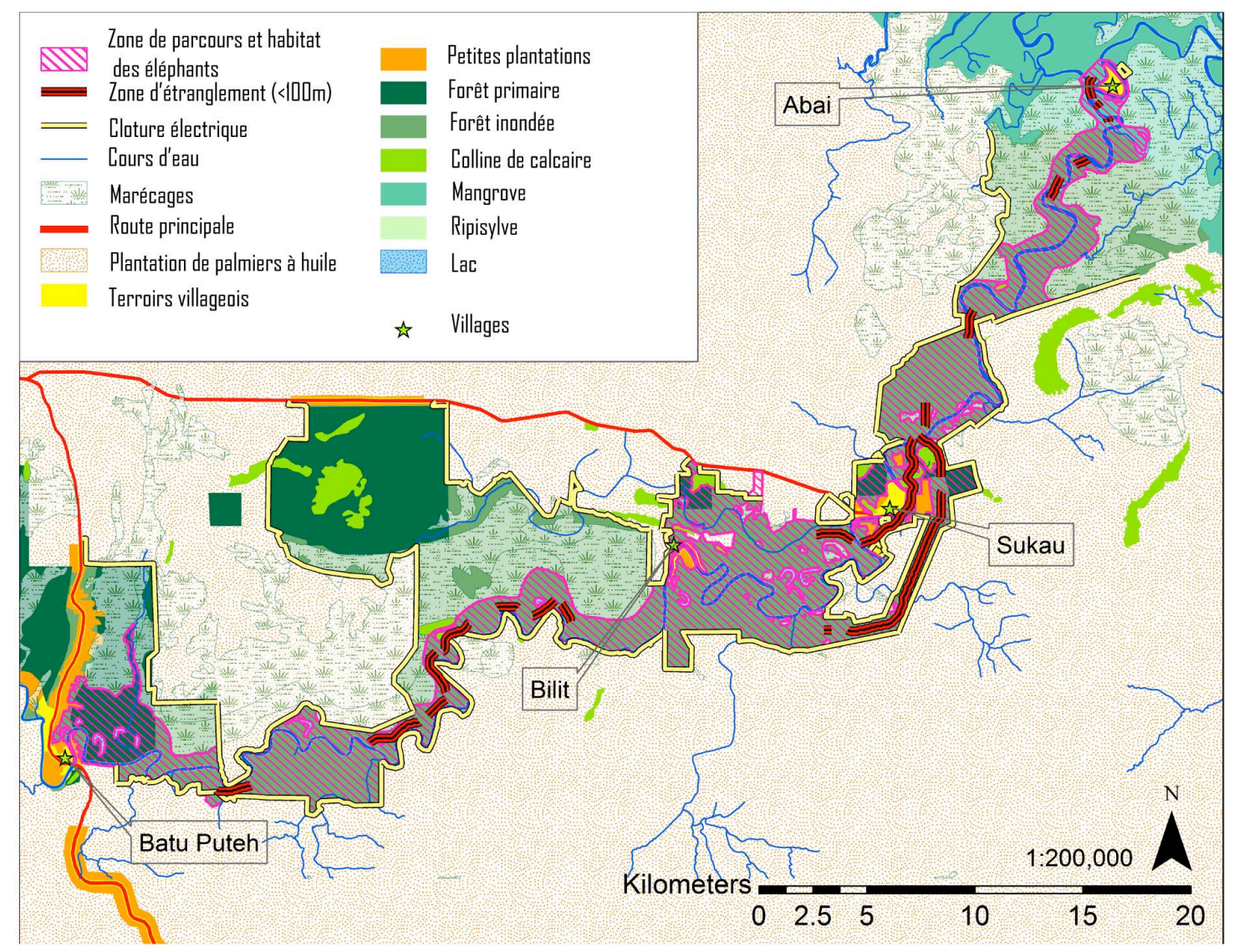




\section{Un territoire humanimal au-delà de la rhétorique du conflit}

Une première approche consiste à lire les relations homme animal sous le prisme du conflit. En effet, la majorité des petits planteurs autochtones et des propriétaires terriens d'origine urbaine considère que la faune est nuisible. Entretenir un potager revient à attirer des singes et des troupeaux de sangliers à barbe (Sus barbatus) qui les saccagent (entretiens Abai, Bilit, Sukau, 2008-2009). Dans la Kinabatangan, entre 1990 et 2000, Lee Shan Kee rapporte 75 conflits avec des plantations, 41 avec des villageois et une dizaine d'éléphants abattus illégalement, les éléphants causant en moyenne 15000 RM de dommages (env. $3000 €$ de l'époque) sur une même plantation. Depuis 1995 le Sabah Wildlife Department s'assure que les espèces protégées ne sont pas abattues mais transportées vers des réserves (Ambu \& Al., $2002: 16$ ). Ne pouvant plus légalement tuer les animaux, les grandes compagnies ont progressivement ceinturé leurs plantations de clôtures électriques. Même si leur efficacité est limitée (figure 4), cette mise en défens a pour effet de fermer le sanctuaire d'un côté et de reporter les nuisances sur les terroirs villageois de l'autre (figure $3)$.

Parallèlement, l'augmentation de la conversion des forêts non protégées en plantations de palmiers à huile (Bissonnette, 2011 :142) force les bêtes à se réfugier dans le sanctuaire (Ancrenaz \& Al., 2005 ; SWD, 2011). La densité y augmente donc régulièrement: les éléphants sont passés de 100 à 300 individus au cours des années 2000 (Estes et Al., 2012), les orangs-outans sont environ 1100 dans le sanctuaire (Ancrenaz \& Al., 2005). Par conséquent, la fréquentation des terroirs villageois par la faune est de plus en plus fréquente. Or, les déplacements des animaux hors du sanctuaire les rendent vulnérables : ils menacent les cultures et suscitent des conflits avec les habitants et les planteurs (SWD, 2011). De plus, les pièges tendus pour le gibier blessent sans distinction, que l'espèce soit protégée par la loi ou non (Sabah State, 1997). 20\% des 300 éléphants du sanctuaire ont des blessures (SWD, 2011 :6). Les ONG et le Sabah Wildlife Department invoquent ces faits pour promouvoir la création d'un couloir végétal continu qui engloberait les villages en élargissant les voies de contournement (Ancrenaz \& Al., 2005 ; SWD, 2011). Le projet du fonds mondial pour la nature fait environ 120 kilomètres d'est en ouest et 10 à 20 kilomètres de large (WWF, 2001) (figure 2). Or, même si ce projet se concrétise, les terroirs villageois seront toujours ouverts à la faune.

En outre, les portes et les barrières n'arrêtent pas les animaux les plus agiles. Nurzhafarina Othman, biologiste, prouve que les éléphants élaborent des stratégies complexes pour franchir les clôtures (figure 4), (Bucknell, 2012). Dans les villages, les singes ouvrent les fenêtres des maisons et mangent la nourriture conservée dans les placards (observations personnelles, Sukau, Avril 2009). Les entretiens révèlent que le problème principal réside dans I' " intelligence " de ces mammifères. La capacité de l'animal à produire des solutions 
pour détruire ou contourner un obstacle complique les modalités de la conservation. Leur comportement force les décideurs à penser des solutions au-delà de la protection des animaux et des limitations des conflits. Il s'agit de créer les modes de cohabitation entre espèces animales et humaines (interview de Marc Ancrenaz, Mongabay, 2010).

Figure 4 : Eléphants franchissant une clôture électrique de façon individualisée (Nurzhafarina Othman, in Bucknell, 2012) :

"Sandy, le plus grand, s'électrocute, se recule, évalue la situation, et prend le temps de franchir lentement la clôture sans la toucher. Le second éléphant, plus petit, trouve un creux qu'il approfondit avec ses défenses pour se faufiler sous la clôture » (ma traduction) (Bucknell, 2012).

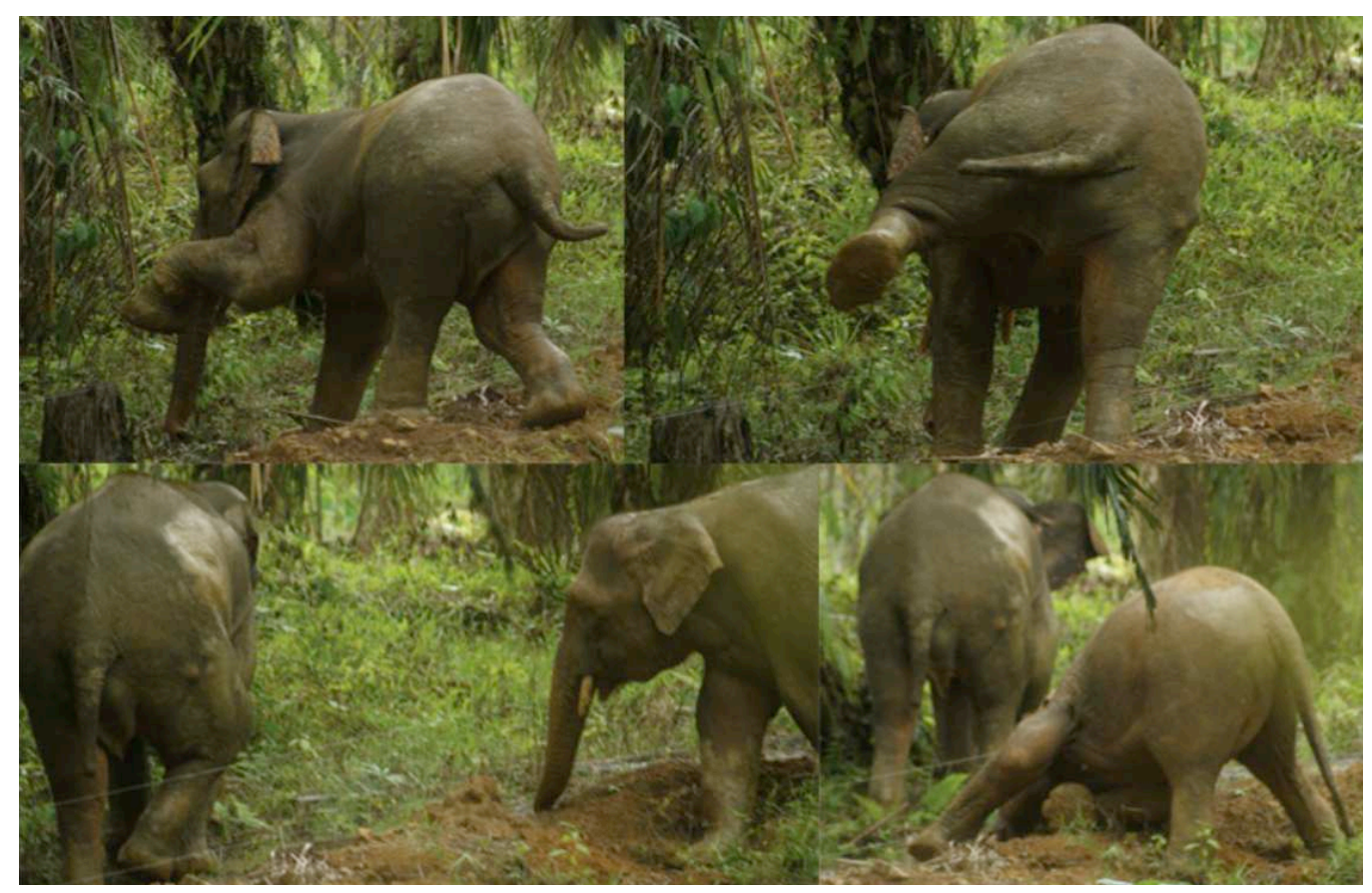

Ce partage de l'espace, qui est un problème pour les habitants, est en revanche un atout pour le tourisme. Or, si les habitants n'acceptent pas la faune, le conflit homme-animal autour des cultures risque de glisser vers un conflit entre habitants et touristes.

Avant la création du sanctuaire, les villageois étaient nombreux à pratiquer le braconnage et à faire des coupes illégales dans les réserves forestières (Vogel, 2005). En 1999, I'un d'eux déclare "Pourquoi les tours opérateurs peuvent-ils gagner de l'argent à nos dépens ? Si nous ne pouvons pas bénéficier du tourisme, nous irons tuer le dernier singe nasique, comme ça, les tours opérateurs n'auront plus rien à montrer à leurs touristes!" (ma traduction) (Schulze et Al., 1999). Le tourisme suscite l'incompréhension chez de nombreux habitants, surtout les plus âgés (Hussin, 2006). Les raisons de coopérer à la protection de l'environnement sont minces. Dans le but de susciter l'adhésion des habitants, Hutan, puis 
WWF prennent en compte l'intérêt des populations sur trois plans : ils offrent des emplois à des villageois (une quarantaine d'emplois permanents chez Hutan, des emplois temporaires chez WWF), tentent d'apaiser les conflits et utilisent le tourisme pour faire accepter leur présence (Lackman, 2003 ; Ancrenaz, 2007). Tout d'abord, Hutan investit dans une clôture électrique pour le cimetière de Sukau dont le saccage régulier est le plus grand sujet de colère chez les habitants. Ensuite, ils développent des systèmes peu coûteux et inoffensifs pour les animaux. Par exemple, ils protègent le tronc des arbres avec des plaques de zinc pour empêcher les orangs-outans d'y grimper et d'en manger les fruits (Lackman, 2003). Un réseau de gardes de la faune, les "wildlife wardens " et un dispositif d'alerte sont créés en 2001 par quelques habitants, des institutions locales, des membres d'ONG et des scientifiques. Quand un troupeau d'éléphants approche, des pétards sont lancés pour les effrayer et les détourner des villages et des plantations. La liberté de déplacement des grands mammifères hors des zones protégées est ici considérée comme une donnée avec laquelle les habitants doivent apprendre à vivre ce qui suscite la constitution de structures englobant plusieurs groupes d'acteurs aux intérêts à priori divergents.

\section{Les récits des rencontres hommes-animaux : l'émergence d'un collectif}

Malgré les désagréments encourus, le regard des habitants sur les bêtes est respectueux. Un villageois de Bilit possédant de petits étangs artificiels, saccagés près d'une fois par an par les éléphants, les désigne sous le nom affectueux de Nenek, grand-parent, et de Raja Binatang, roi des animaux. II personnifie leur comportement en expliquant qu'ils aiment jouer dans l'eau et qu'il est impossible de les faire fuir car "ils n'ont pas peur et préfèrent

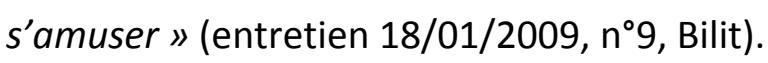

Ce discours est révélateur de l'attitude de nombreux habitants. Au cours d'épisodes dramatiques comme les inondations de février 2010, la vie du collectif homme-animal devient visible. "Je pense que la rencontre la plus inoubliable a été celle d'un adulte orang outan qui était coincé en haut d'un arbre en raison de la crue (...) il était déjà là depuis plusieurs jours et (...) les villageois lui avaient donné de la nourriture pour l'aider " (courriel 2012, touriste anglophone, cadre, séjour janvier février 2010). Au-delà de l'anecdote valorisante pour le touriste qui la raconte, c'est une manifestation de la relation riche et complexe entre humains et animaux, spécifique à la plaine alluviale de la Kinabatangan. Celle-ci semble jouer un rôle dans l'atténuation des conflits hommes-animaux.

Cette attitude rejoint la proximité symbolique existant entre les habitants et leur milieu. Des légendes du Sabah mettent en scène un collectif homme-animal. Elles font référence " au temps où les animaux parlaient " (ma traduction), leurs héros sont des hommes et des bêtes qui appartiennent à la même fratrie. Selon une légende de la basse Kinabatangan, les crocodiles de rivière descendent des humains et respectent les orang sungai. Ils ne les attaquent pas et ceux-ci les respectent (Shim, 2002 : 50). Malgré ce pacte symbolique, les 
habitants pêchent toujours en bateau et quand ils se baignent dans la rivière, ils restent peu dans l'eau. Les dernières personnes qui ont été attaquées par des crocodiles sont des travailleurs immigrés qui pêchaient dans un lac, le corps à moitié immergé dans l'eau (terrain, 18/05/09). La proximité romancée existant entre les sociétés et la faune locale se double d'habitudes qui consistent à ne pas s'exposer aux risques représentés par les prédateurs. Cet ensemble favorise leur cohabitation en minimisant les conflits hommeanimal.

Le collectif "humanimal » actuel hérite également de pratiques anciennes. Des vergers plantés par les habitants, mais passés inaperçus aux yeux des premiers explorateurs, enrichissent la jungle en aliments comestibles. Cette particularité de l'écosystème local explique en partie pourquoi les primates survivent malgré les fortes densités rencontrées (entretien avec I. Lackman 10/01/2008). La " capacité de charge » du sanctuaire a donc été augmentée grâce à l'action humaine.

En outre, les habitants, musulmans dans leur immense majorité, respectent de nombreuses espèces en raison des interdits de leur religion. L'Islam les autorise à chasser le cerf sambar (cervus unicolor) mais la viande des primates, celles des éléphants bien d'autres encore, est strictement interdite (Boubakeur, 2001 ; entretiens). Ces interdits religieux sont invoqués par les habitants qui se présentent comme responsables de la forte densité actuelle de primates dans leur région (entretiens).

L'évolution du collectif société-animaux a donc donné naissance au paysage actuel. L'irruption du tourisme revalorise rétroactivement ces liens. Comme le dit le chef du village de Bilit : "la forêt est vraiment belle, s'il n'y a pas de forêt, il n'y a pas de touristes " (Bilit, Janvier 2009). Pour un homme dont le fils travaille en collaboration avec WWF et dont plusieurs enfants et neveux sont employés dans le tourisme, l'utilité de la jungle comme produit touristique est évidente.

\section{La mise en scène de la rencontre homme-animal par les opérateurs touristiques}

La faune étant libre de se déplacer où bon lui semble à l'intérieur voire à l'extérieur du sanctuaire, chaque séjour comporte le risque de voir peu d'animaux iconiques tels que les orangs-outans et les éléphants. Comme le relate un touriste au sujet des éléphants "les guides sont généralement de type à prévenir les touristes que la rencontre de l'animal (...) est possible mais qu'il ne faut pas y compter» (courriel, 2012, cadre français, séjour en septembre 2006). Pour voir des orangs-outans, ils leur conseillent d'aller au centre de réhabilitation de Sepilok; des itinéraires intègrent d'ailleurs les deux destinations (figure 1). Les guides gratuits édités par le Sabah Tourism Board ne mentionnent pas les orangs-outans et les éléphants dans les pages concernant la Kinabatangan (STB, 2005). Celle-ci est présentée comme l'habitat des singes nasiques, des calaos et autres oiseaux car ils sont 
beaucoup plus faciles à voir. Et pourtant, comme le déclare un touriste : " mon objectif en venant sur l'ile de Bornéo était avant tout d'avoir la chance d'observer l'orang-outan dans son milieu naturel, mais sans connaitre avec précision cet animal (...). J'étais également très désireux de visiter la jungle et son ambiance si particulière(...) Je n'étais pas franchement [intéressé] de voir les animaux dans des parcs type Sepilok» (courriel 2012, touriste français, cadre, séjour en juin 2008). En effet, les touristes nomment l'orang-outan en tête des espèces qui motivent leur visite dans la Kinabatangan (entretiens auprès de 73 touristes qui ont visité la zone d'étude, mai 2009). Les opérateurs ont deux choses à gagner en présentant le risque de ne pas voir beaucoup d'animaux. D'une part, comme la satisfaction des touristes est étroitement liée à la rencontre de l'un de ces mammifères (Chan et Al., 2007), les touristes seront moins déçus. D’autre part, ils peuvent vendre des séjours de trois jours et deux nuits, au lieu d'une nuit. Avec les quatre croisières sur la rivière, les deux marches de jour et deux marches de nuit, les chances d'admirer la faune sont doublées ainsi que les profits des tours opérateurs, dans une optique gagnant-gagnant.

L'espace qui prend de la valeur est là où la rencontre homme-animal peut être maitrisée. Dans la Kinabatangan, cela correspond majoritairement au fleuve qui offre une discontinuité dans l'espace semi-opaque de la forêt où la faune est difficile à discerner. Sur la terre ferme, la lisière de la forêt et les lacs offrent des ruptures dans le couvert végétal. C'est près de ces discontinuités que les opérateurs cherchent à construire les hébergements.

La liberté d'action et de déplacement des bêtes est mise en scène par les opérateurs touristiques. Ils reprennent les habitudes des villageois en plantant des arbres fruitiers, non pas pour leurs besoins alimentaires mais pour attirer les animaux. Au lodge d'Abai en août 2010, les touristes ont pris leur petit déjeuner dehors en admirant les circonvolutions d'une femelle orang-outan dans les arbres situés au-dessus de leurs assiettes. Le soir, un écureuil volant a fait une démonstration de vol plané entre les bungalows. Après le dîner, un loris paresseux de Bornéo a attiré tous les convives auprès d'un petit bosquet situé au centre des bungalows.

Pour maximiser les possibilités de rencontre, les lodges se dotent de passerelles de bois qui surplombent le sol marécageux et offrent ainsi un parcours de promenade à l'abri des crocodiles. En termes de conservation, la plate-forme fixe semble plus respectueuse de la liberté de l'animal. Les touristes aiment constater que c'est l'animal qui s'approche d'eux, "librement ». Au contraire, la plate-forme mobile constituée par le bateau effectue une intrusion sur le territoire de l'animal où l'homme maîtrise la distance (Higham, 2008). L'animal peut intimider les visiteurs comme le relate ce touriste au sujet d'une ballade en barque : « il [l'orang-outan] ne fût pas content de nous voir, [...] il nous a lancé des baies, des branches [...] pour tenter de nous effrayer et de nous faire fuir !» (courriel 2012, touriste anglophone, cadre, séjour Batu Puteh, janvier-février 2010). Parfois des touristes se sentent 
trop près des animaux et se plaignent au guide (observations personnelles, Abai, 2010). En revanche, d'autres proposent de l'argent pour inciter le guide à se rapprocher, ce que les professionnels ont pour consigne de refuser (entretiens Sukau, décembre 2007). La conception de la distance idéale varie selon le type de plate-forme, la perception du danger, le désir de faire une bonne photo mais aussi l'attitude de l'animal.

Des discours compensateurs sont aussi utilisés. A une touriste qui n'a vu ni éléphants, ni orang-outans, le guide de Batu Puteh lui indique qu'elle a vu plusieurs espèces de calaos en insistant sur la rareté du phénomène (entretien informel 27 mars 2012). Voici comment elle relate son expérience par courriel: "Et alors que la promenade s'axait sur la recherche de l'orang outang qui resta caché, j'ai été surprise par la diversité des animaux qui m'entourent, et qui, sans l'œil avisé de mon guide, me seraient restés invisibles. Si l'observation des singes étranges que sont les nasiques et le rire qu'a provoqué la voltige des macaques étaient le plus recherché mais aussi le plus accessible, mon guide $m^{\prime}$ a aidé à prendre conscience de la chance que j'avais de voir des spécimens d'oiseaux rares» (courriel 2012, étudiante française, séjour avril 2012). Pourtant, les observations menées lors d'autres croisières dévoilent que, quand les orangs-outans ou les éléphants sont en vue, les guides s'arrêtent à peine devant les calaos et les nasiques. Dans ce cas, il est clair que les guides parviennent subtilement à prévenir la déception de la touriste en insistant sur des particularités qu'ils ne mentionnent pas quand les espèces les plus charismatiques sont présentes.

Figure 5 : Démonstration de l’intelligence pratique d'une éléphante nageant en aval de son petit. (Photo de l'auteur : Septembre 2006)

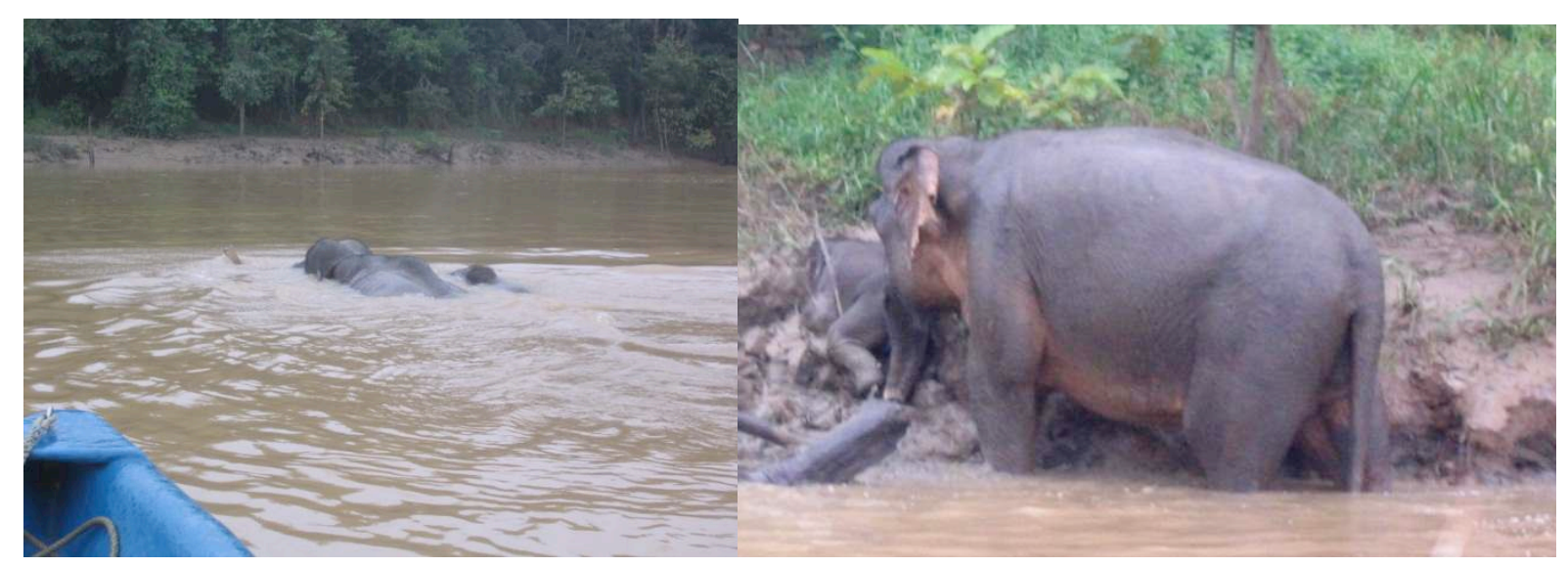

L'intelligence pratique des animaux fait sensation. "Oh, il utilise un bâton... c'est génial " s'extasie une touriste qui admire un macaque cherchant à capturer un crabe (observations personnelles, Août 2010). Lors d'une croisière à Bilit, l'ingéniosité d'une mère éléphant pour protéger son petit rend les voyageurs béats. "La vue lors du deuxième jour d'une femelle traversant la rivière ainsi que sa façon de protéger son petit du courant m'a rendu complètement frénétique (...) de même que les autres touristes dans l'embarcation, et [a] 
ainsi laissé un souvenir indélébile dans mon esprit (...). Cet instant a peut-être été le moment le plus marquant du séjour en Malaisie» (cadre supérieur français, séjour en septembre 2006). En effet, la mère nageait en aval ce qui empêchait que l'éléphanteau ne soit emporté par le courant (Bilit, septembre 2006). La traversée du fleuve par les éléphants est le point culminant du séjour pour beaucoup de touristes, or, non seulement c'est un signe de I'insuffisance de la taille du sanctuaire mais en plus, c'est un facteur de stress pour les pachydermes (Estes et Al., 2012).

La fascination exercée par les animaux sur les touristes a donné naissance à Red Ape Encounter, le tour opérateur villageois de Sukau, monté avec le soutien de Hutan. Chaque année, un maximum de trois-cents touristes passionnés par les primates s'offre une expérience en éthologie appliquée. Ils ont l'autorisation d'accompagner des assistants de recherche dans un site scientifique situé dans le sanctuaire. Ils suivent les orangs-outans dans leurs déplacements et décrivent leur comportement par écrit. Ils repèrent les outils utilisés pour la construction des nids, les modes d'interactions sociales, la nourriture choisie etc. Les primates sont désignés par un prénom et les touristes qui participent à cette expérience expriment une sorte d'attachement pour eux (entretiens, Sukau 2009). La présence humaine cause un niveau de stress moins élevé aux orangs-outans fréquentant le site qu'à leurs comparses (Muehlenbein, 2012).

L'habituation des primates signe une perte du "sauvage " souvent considérée comme négative (Shelton et Al. 2007), or il s'agit peut-être d'une évolution caractérisant une nouvelle forme de collectif homme-animal incluant les touristes et redéfinissant les paradigmes de la cohabitation entre la faune et la société locale à moyen terme, ceci, en raison du changement de comportement des orangs-outans et du regard des habitants. En effet, certains habitants adoptent parfois un comportement similaire à celui des touristes. A plusieurs reprises, nous avons vu des éléphants pénétrer dans les jardins des homestays où nous logions, les parents sont allés chercher les enfants, et se sont postés à une fenêtre pour profiter du spectacle en famille, sans chercher à les faire fuir (observations personnelles, Bilit, 2008 et 2009).

\section{Conclusion}

Le sanctuaire est un espace refuge dans lequel l'augmentation de la densité des mammifères est bénéfique au tourisme. Dans le cadre de leur mise en scène de la nature, les opérateurs touristiques sont ceux qui ont le plus intérêt à ce que les villageois ne s'opposent pas à la proximité des bêtes sauvages. En retour, c'est grâce à ce secteur qu'une partie de la population accepte les animaux et les mesures de protection de l'environnement. 
Les acteurs locaux et les touristes attribuent aux animaux un statut d'individus libres et intelligents, dont le comportement est source d'admiration. Or, ces caractéristiques leur permettent de contourner les obstacles et force les groupes humains à redéfinir les modes de préservation des espèces et de cohabitation entre habitants et animaux. En tant qu'acteurs, les animaux suscitent donc de nouvelles alliances entre institutions, habitants, ONG environnementalistes, scientifiques et opérateurs touristiques comme le montre la création de nouvelles associations telles que les gardes de la faune, le tour opérateur villageois Red Ape Encounter, le homestay etc... La fréquentation des espaces villageois par les animaux réactive les symboles du collectif homme-animal hérités d'une période où les animaux sauvages étaient hors des villages. Progressivement, les sociétés et la faune locale inventent un nouveau collectif homme-animal.

\section{Bibliographie}

Ambu L. N., Andua P. M., Nathan S., Tuuga A., Jensen S. M., Cox R., Alfred R., Payne J., (2002) Asian elephant action plan Sabah, Sabah Wildlife Department, Kota Kinabalu, [en ligne] http://www.wildlife.sabah.gov.my/Last\%20ed\%20of\%20Elephant\%20strategy.pdf

Ancrenaz M., Gimenez O., Ambu L., Ancrenaz K., Andau P., Goossens B., Payne J., Tuuga A., LackmanAncrenaz I. (2005) " Aerial survey gives new estimates for orang-utans in Sabah, Malaysia». Public Library of Science (PloS) Biology 3(1): 30-37.

Ancrenaz M. (2007) «The Costs of Exclusion: Recognizing a Role for Local Communities in Biodiversity Conservation", in PLoS Biol, v.5 (11); Nov 2007, 8p.

Baratay E. (2010) "Les socio anthropologues et les animaux, Réflexions d'un historien pour un rapprochement des sciences ", Sociétés 108, 2 (2010) 9-18.

Bernard S., Bissonnette J.F. (2011) " Oil plantations in Sabah: Agricultural Expansion for whom? » in De Koninck R., Bernard S., Bissonnette J.-F., Borneo transformed, agricultural Expansion on the Southeast Asian Frontier, CHATSEA, NUS Press, Singapore, pp. 120-151.

Boubaker D. (2001) Halal \& Haram, le licite et l'illicite alimentaire en Islam, Institut Musulman de la Mosquée de Paris, 68 p. accès[ en ligne] www.mosquee-de-paris.net/Conf/Halal.pdf

Bucknell D. (2012) "Overcoming challenges to save Bornean elephants», in Elephant Family, 16th August 12, [en ligne] http://www.elephantfamily.org/what-we-do/conservation-news/overcomingchallenges-to-save-bornean-elephants/

Chan J. \& Baum T. (2007) " Determination of satisfiers and dissatisfiers Using Herzberg's motivator and Hygiene Factor Theory : an exploratory study ", in Tourism, culture and communication, Vol. 7 Number 2, 2007, pp. 117-132.

Dalla Bernardina S. (1996) L'utopie de la nature, chasseurs, écologistes et touristes, Imago, Paris, $304 p$. 
Dalla Bernardina S. (2006) L'éloquence des bêtes: Quand I'homme parle des animaux, Editions Métailié, 200p.

De Koninck R. (2007) Malaysia: La dualité territoriale, Belin, 190p.

Dove, M., Sajise P., et Doolittle A. (dir.) (2005) Conserving Nature in Culture: Case Studies From Southeast Asia, Yale Univ, Southeast Asia Studies, 368p.

Espaces et Sociétés, $\mathrm{n}^{\circ}$ 110-111, (2002) la place de l'animal [en ligne] http://www.espacesetsocietes.msh-paris.fr/110111/sommaire.html

Estebanez J. (2006) " Les jardins zoologiques et la ville: quelle nature pour le biodôme de Montréal? ", in Annales de géographie 6/2006 ( $\mathrm{n}^{\circ}$ 652), p. 708-731. [en ligne]: www.cairn.info/revue-annales-de-geographie-2006-6-page-708.htm.

Estes JG., Othman N., Ismail S., Ancrenaz M., Goossens B., et al.(2012) "Quantity and Configuration of Available Elephant Habitat and Related Conservation Concerns in the Lower Kinabatangan Floodplain of Sabah, Malaysia», in PLOS ONE 7(10), [en ligne] http://www.plosone.org/article/info\%3Adoi\%2F10.1371\%2Fjournal.pone.0044601

Godet, L. (2010) " la nature ordinaire dans le monde occidental ", in L'Espace géographique, 2010/4 - Tome 39, pp. 295-308

Goossens B., Ambu L., "Sabah Wildlife Department and 10 years of research: towards a better conservation of Sabah's wildlife», in Journal of Oil Palm and the Environment, 2012, 3, pp. 38-51.

Gunnel Y. (2009) Ecologie et société, Armand Colin, Paris, 415 p.

Héritier S. (2008) " Gestion du territoire "parc », tourisme et communautés locales », in Héritier S., Laslaz L. (dir.), Les parcs nationaux dans le monde : protection, gestion et développement durable, Ellipses, Paris, 267-288p.

Higham J., Lusseau D., Hendry H. (2008) «Wildlife viewing: the significance of the viewing platforms. In Journal of Ecotourism, Vol. 7 n 2 \& 3, pp. 132-141

Hingginbottom, K. (2004) Wildlife tourism, Impacts, management and planning, Sustainable Tourism, Publisher-site.com, Common Ground Publishing, Altona vic, 277p., [en ligne] http://www.sustainabletourismonline.com/117/wildlife-tourism/wildlife-tourism-impactsmanagement-and-planning

Houston zoo (2012) [en ligne] http://www.houstonzoo.org/animalart/

Hutton W. (2001) Discovering Sabah, Natural History Publications, Kota Kinabalu, 177p.

HUSSIN Rosazman (2006) Ecotourism development and local community participation : case studies of Batu Puteh and Sukau village in Lower Kinabatangan area of Sabah, Malaysia, Thesis, Department of Sociology, Anthropology and applied social sciences, University of Glasgow, UK, 454p. 
Lackman I. (2003) "Community-Based Conservation Project in Kinabatangan, Sabah» HUTAN in Mohamed M., Kusano T., (Eds) Effective Approaches to Nature Conservation; Proceedings of the BBEC International Conference 2003, 25th - 27th, February 2003, Tuaran, Sabah, Malaysia pp. 42-45

Lee Shan KHEE (2002) Human elephant conflict in lower Kinabatangan, Sabah, Master Thesis, School of science and technology, University Malaysia Sabah, Kota Kinabalu 70p.

Lestel, D. (2001) Les origines animales de la culture, Flammarion, 414p.

Lussault M. (2007) L'homme spatial : la construction sociale de l'espace humain, Seuil, Paris, 364 p.

Mauz I. (2002) "Les conceptions de la juste place des animaux dans les Alpes françaises ", In Espaces et Sociétés, $n^{\circ} 110-111,2002$ pp 129-147

Mauz I. (2005) Gens, cornes et crocs, INRA, CEMAGREF, CIRAD, IFREMER, 255p.

Michaud M. (2011 a) Chasseurs d'Afrique Safari de chasse et quête du sauvage, (Vol. I), Thèse de doctorat, Université de Lyon II, 359p.

Michaud M. (2011 b) "Acheter un éléphant sur le web, la commercialisation des safaris sur Internet ", in Cros M et Mégret Q., Net et terrain, ethnographie de la nature en Afrique, Editions des archives contemporaines, Paris, pp. 49-65

Mongabay.com (2010) «Orangutans vs palm oil in Malaysia: setting the record straight. Interview by HUTAN's Dr. Marc Ancrenaz», January 16, 2010 [en ligne] http://news.mongabay.com/2010/0116orangutans.html

Mounet C. (2007) Les territoires de l'imprévisible, Conflits, controverses et "vivre ensemble" autour de la gestion de la faune sauvage. Le cas du loup et du sanglier dans les Alpes françaises, Thèse de doctorat, 564p., [en ligne] http://tel.archives-ouvertes.fr/tel-00207766/en/

Mowforth M \& Munt I.(2008) Tourism and Sustainability: Development, Globalisation and New Tourism in the Third World, $3^{\mathrm{e}}$ éd., Routledge, $424 \mathrm{p}$.

Muehlenbein MP. \& al. (2012) «Ape Conservation Physiology: Fecal Glucocorticoid Responses in Wild Pongo pygmaeus morio following Human Visitation», [en ligne] http://www.plosone.org/article/info\%3Adoi\%2F10.1371\%2Fjournal.pone.0033357

Padoch C., Peluso N., \& Danks L. (2003) Borneo in Transition: People, Forests, Conservation, and Development, Oxford University Press, $346 \mathrm{p}$.

Payne J. (1989) A tourism feasibility study for the proposed Kinabatangan wildlife sanctuary, WWF Malaysia, Kuala Lumpur, 51p. [en ligne] http://repository.wwf.org.my/technical_reports/A/ATourismFeasibilityStudyForTheProposedKinabat anganWildlifeSanctuary.pdf

Revolinski K. (2011) " 10 awesome wildlife destinations ", in Reader's digest, 14/02/2011, accédé le 12 Avril 2012, [ en ligne] http://www.readersdigest.ca/world-travel/10-awesome-wildlife- 
Sabah State (1997) Sabah Wildlife Enactment, Kota Kinabalu, 89p. [en ligne] http://www.wildlife.sabah.gov.my/Enactment.htm

Sabah State (2005) " Declaration of a wildlife sanctuary at district of Kinabatangan and Sandakan under section 9», Wildlife conservation enactment 1997, Government Gazette, Sabah Malaysia, 11 $08 \quad 2005, \quad n^{\circ} \quad 31$, (No KPKAS:(R) 100-52/1 Klt.7). [en ligne] http://www.sabah.gov.my/gazette/docs/001801.pdf

Sabah Wildlife Department (SWD) (2011) Elephant Action Plan, Kota Kinabalu, Sabah, Malaysia.

Schulze H., Suratman S. (1999) «Ecotourism development in the Lower Kinabatangan area: the perspective of the Orang Sungai» in Villagers in Transition: Case Studies from Sabah. Universiti Malaysia Sabah, pp. 4-6.

Sellato B. (1998) «Forêts tropicales et sociétés traditionnelles à Bornéo, vers une histoire en continu des sociétés et des systèmes de subsistance», in Cahiers d'Outre-mer $n^{\circ} 51$, Sept-Oct 1998, pp. 421439

Shelton J., Higham J. (2007) "Ecotourism and wildlife habituation», Higham (Ed), Critical issues in Ecotourism, understanding a complex tourism phenomenon, Butterworth Heinemann, pp 271-286.

Shim P.S. (2002) Animal tales of Sabah, Natural History Publication, Kota Kinabalu, Sabah.

Van Schaik C., Ancrenaz M. (\& Al.) (2003) «Orang-utan Culture and the Evolution of Material culture», in Science 3, Vol. 299, no. 5603, pp. 102 - 105

Vogel M., Abd Hamid M. (2005) «Developing Community based tourism at the Kinabatangan basin: a review of the Mescot Project», presented at International conference Pro Poor tourism: Mechanism and Mainstreaming, Melacca, 12p.

WWF Malaysia (2001) A corridor of Life, a vision for the Kinabatangan 2020, Partners for Wetlands, Kota Kinabalu, 22p.

Zeppel H., Muloin S. (2008) "Aboriginal interpretation in Australian Wildlife tourism", Journal of Ecotourism, Vol 7, n² \&3, 2008, pp. 111-131. 